\title{
Dynamical response of the Galileo Galilei on the ground rotor to test the equivalence principle: Theory, simulation, and experiment. II. The rejection of common mode forces
}

\author{
G. L. Comandi \\ Istituto Nazionale di Fisica Nucleare (INFN), Sezione di Pisa, Largo B. Pontecorvo 3, I-56127 Pisa, Italy and \\ Department of Physics, University of Bologna, Bologna, Italy \\ R. Toncelli \\ Istituto Nazionale di Fisica Nucleare (INFN), Sezione di Pisa, Largo B. Pontecorvo 3, I-56127 Pisa, Italy \\ M. L. Chiofalo \\ Scuola Normale Superiore, Piazza dei Cavalieri 7, I-56100 Pisa, Italy and Instituto Nazionale di Fisica \\ Nucleare (INFN), Sezione di Pisa, Largo B. Pontecorvo 3, I-56127 Pisa, Italy \\ D. Bramanti \\ Istituto Nazionale di Fisica Nucleare (INFN), Sezione di Pisa, Largo B. Pontecorvo 3, I-56127 Pisa, Italy \\ A. M. Nobili \\ Department of Physics “E. Fermi," University of Pisa, Largo B. Pontecorvo 3, I-56127 Pisa, Italy
}

(Received 5 January 2006; accepted 16 January 2006; published online 23 March 2006)

"Galileo Galilei on the ground" (GGG) is a fast rotating differential accelerometer designed to test the equivalence principle (EP). Its sensitivity to differential effects, such as the effect of an EP violation, depends crucially on the capability of the accelerometer to reject all effects acting in common mode. By applying the theoretical and simulation methods reported in Part I of this work, and tested therein against experimental data, we predict the occurrence of an enhanced common mode rejection of the GGG accelerometer. We demonstrate that the best rejection of common mode disturbances can be tuned in a controlled way by varying the spin frequency of the GGG rotor. (C) 2006 American Institute of Physics. [DOI: 10.1063/1.2173076]

\section{INTRODUCTION}

The relevance of equivalence principle (EP) tests as the most sensitive probe of general relativity has been strongly motivated from a theoretical point of view. ${ }^{1,2}$ In Part I of this work we have discussed the motivation behind the Galileo Galilei on the ground (GGG) experiment for testing the EP at $1 \mathrm{~g}$ with macroscopic $(10 \mathrm{~kg})$ concentric test cylinders in rapid rotation. The instruments which have provided the best EP tests to date are rotating torsion balances, ${ }^{3,4}$ their essential features being the differential nature of the instrument (i.e., its capability to reject common mode effects) and the modulation of the signal through rotation. It has also been established that very high accuracy tests can be achieved only by performing an experiment in space, inside a spacecraft orbiting the Earth at low altitude. ${ }^{5-7}$ The GGG experiment embodies the key features of the rotating torsion balances, with the addition of being suitable for flight.

The GGG experiment ${ }^{8,9}$ has been described in Part I, and its underlying physics has been embodied in an effective model that fully accounts for the measured normal modes of the GGG rotor in the whole range of spin frequencies, from subcritical to supercritical rotation.

Here, we apply the model to evaluate the common mode rejection capability of the GGG rotor as determined by all the system parameters which govern the design of the instru- ment. This study naturally provides an effective tool to optimize the real instrument in response to external disturbances such as tidal forces ${ }^{10}$ and seismic noises. ${ }^{11}$

We refer to Part I for all the definitions and the description of the experiment, as well as of the model. This Part II is organized as follows. In Sec. II the numerical method developed in Part I is completed by including external forces, in common mode and in differential mode. In Sec. III, we compute the common mode rejection factor, first at zero spin, through an analytical solution depending on one scaling parameter, and then in rotation, through our numerical simulation model; numerical simulations show the relevance-in wide ranges of the spin frequency — of the analytical scaling parameter and demonstrate the existence of an enhanced common mode rejection. In Sec. IV we apply these results to the realistic range of parameters of the GGG rotor and discuss how the enhanced rejection of common mode effects can be exploited for optimizing the performance of the instrument in testing the equivalence principle. Concluding remarks and perspectives after both Parts I and II are given in Sec. V.

\section{THE NUMERICAL METHOD}

\section{A. Dynamical equations: External forces and transfer function}

In Sec. IV of Part I we have discussed the numerical simulation method of the model used to describe the GGG 
instrument and the parameters of the system (see Figs. 1 and 3 of Part I for the GGG instrument and its model). Here we describe the transfer matrix method, in the presence of external forces-acting on the system in common mode as well as in differential mode- which determine the dynamical behavior of the rotor. External forces are added to the right-hand side of the equations of motion, written as in Eq. (37) of Part I, which now becomes

$$
\dot{X}=A X+B U,
$$

where $A$ is the $2 n \times 2 n$ dynamical matrix already appearing in Eq. (38) of Part I, $X$ is the vector of generalized coordinates and velocities defined in Eq. (36) of Part I, while the $2 n \times m$ input matrix $B$ and the input vector $U$ have been added, the $m$ components of $U$ representing the external forces. The definition of the problem is completed after specifying the $p$ component output vector $Y$ by means of the general relationship

$$
Y=C X+D U,
$$

where $C$ is the $p \times 2 n$ output matrix and $D$ is the $p \times m$ input-output coupling matrix. In our problem, $D=0$ and the $Y$ 's are the displacements of the masses from their equilibrium positions.

Equations (1) and (2) are solved in the frequency domain, after Laplace transform to the variable $s=i \omega$. By combining them into a single equation, we have the direct link between the output vector and the input forces,

$$
Y(s)=C(s I-A)^{-1} B U(s) \equiv H(s) U(s) .
$$

Equation (3) defines the $p \times m$ transfer matrix $H$, in the rotating reference frame, in terms of the matrices $A, B$, and $C$ ( $I$ is the identity matrix). The derivation of matrices $C$ and $B$ is given in the Appendix.

The poles $p_{r}$ and the zeros $z_{r}$ of the transfer matrix fully determine the dynamical response of the rotor: the poles are located at the excitation energies, and the zeros tell us where external effects are suppressed.

The signal of an EP violation would be a relative displacement of the GGG test cylinders in the nonrotating reference frame of the laboratory. Therefore, we need to transform the output vector given by (3) in the rotating frame into the $Y^{\mathrm{NR}}(s)$ displacement vector in the nonrotating laboratory frame. We show in the Appendix how the transfer matrix and thus the output are transformed into the nonrotating frame. This obviously results into shifting the poles from $p_{r} \pm i \omega_{s}$ to $p_{r}$ and $p_{r}+2 i \omega_{s}$, namely, to zero and twice the angular spin frequency $\omega_{s}$. The latter behavior, expected in the nonrotating frame, has already been outlined in Fig. 4 of Part I (also reported in Fig. 3 of Ref. 8) where the normal modes of the GGG rotor are given as functions of the spin frequency $\nu_{s}$ $=\omega_{s} / 2 \pi$, showing the existence of horizontal normal mode branches and of inclined ones (at $2 \nu_{s}$ angle), as well as the presence of three instability regions at values of the spin frequency which are resonant with the three natural frequencies of the GGG system.

In the GGG setting reported here the values of the natural frequencies are $\nu_{D} \simeq 0.09 \mathrm{~Hz}$ for the differential one and $\nu_{C 1} \simeq 0.9 \mathrm{~Hz}$ and $\nu_{C 2} \simeq 1.26 \mathrm{~Hz}$ for the two common mode ones. The normal mode behavior defines two main spin frequency regions. One region-that we call the region of "intermediate spin frequencies"-is where $\left|p_{r} \pm 2 i \omega_{s}\right|<\left|p_{r}\right|$ or $\left|z_{r} \pm 2 i \omega_{s}\right|<\left|z_{r}\right|$ (i.e., $0.09<2 \nu_{s}<1.26 \mathrm{~Hz}$ in our case). The other region-that we call the region of "low and very high spin frequencies"-is where $\left|p_{r} \pm 2 i \omega_{s}\right|>\left|p_{r}\right|$ and $\left|z_{r} \pm 2 i \omega_{s}\right|$ $>\left|z_{r}\right|$, namely, on either side of the intermediate frequency region. As we have seen in Part I, the intermediate frequency region is where mode crossings occur; we therefore expect that in this region the rejection of common mode forces will depend very much on the particular frequency at which the system is spinning, while it should not be so in the region of low and high frequencies. The rejection of common mode forces will depend on the frequency region.

\section{RESULTS}

\section{A. The common mode rejection factor}

In this section we define and evaluate the common mode rejection factor $\chi$ which describes the rotor's capability, as a differential instrument, to reject common accelerations as compared to those acting in a differential manner on the test bodies. The smaller the rejection factor $\chi$, the better the performance of the instrument. The rejection is a function of the frequency $\nu$ of the external force applied, as it is the dynamical response of the system.

We have proceeded to evaluate numerically $\chi(\nu)$ by first determining the transfer function in the rotating reference frame for the two cases of common and differential accelerations acting on the test cylinders. The common $H_{C}^{\mathrm{NR}}$ and differential $H_{D}^{\mathrm{NR}}$ transfer functions are then calculated in the nonrotating frame, yielding the corresponding relative displacements $\left\{\Delta x_{C}^{\mathrm{NR}}, \Delta y_{C}^{\mathrm{NR}}\right\}$ and $\left\{\Delta x_{D}^{\mathrm{NR}}, \Delta y_{D}^{\mathrm{NR}}\right\}$ in the $X^{\prime}$ and $Y^{\prime}$ directions of the nonrotating, horizontal plane of the laboratory. It is worth stressing that we are always computing displacements of the test cylinders relative to one another, also in response to an external force acting in common mode; this is precisely because we wish to quantitatively establish how far is our actual instrument from being an ideal differential accelerometer which would give no relative displacement of the test cylinders in response to common mode forces. The relative displacements resulting in both directions of the horizontal plane and depending on the nature of the applied force (either common mode or differential mode) are

$$
\begin{aligned}
& {\left[\begin{array}{l}
\Delta x_{C}^{\mathrm{NR}}(s) \\
\Delta y_{C}^{\mathrm{NR}}(s)
\end{array}\right]=H_{C}^{\mathrm{NR}}\left(s-i \omega_{s}\right) \frac{1}{m_{i}}\left[\begin{array}{c}
F_{X}(s) \\
F_{Y}(s)
\end{array}\right],} \\
& {\left[\begin{array}{c}
\Delta x_{D}^{\mathrm{NR}}(s) \\
\Delta y_{D}^{\mathrm{NR}}(s)
\end{array}\right]=H_{D}^{\mathrm{NR}}\left(s-i \omega_{s}\right) \frac{1}{2 m_{i}}\left[\begin{array}{c}
F_{X}(s) \\
F_{Y}(s)
\end{array}\right] .}
\end{aligned}
$$

The factor $1 / 2$ in (5) is introduced because in this way, if $a_{C}=F / m_{i}=F / m_{o}$ is the acceleration acting in a common manner on the two masses, the differential accelerations are $a_{D i}=F /\left(2 m_{i}\right)$ and $a_{D o}=-F /\left(2 m_{o}\right)=-a_{D i}$, and then $\Delta a \equiv a_{i}$ $-a_{o}=F / m_{i}$.

The rejection factors along the $X^{\prime}$ and $Y^{\prime}$ directions of the plane (not rotating) are therefore defined as follows: 


$$
\begin{aligned}
& \chi_{X^{\prime}}(s)=\frac{\Delta x_{C}^{\mathrm{NR}}(s)}{\Delta x_{D}^{\mathrm{NR}}(s)}, \\
& \chi_{Y^{\prime}}(s)=\frac{\Delta y_{C}^{\mathrm{NR}}(s)}{\Delta y_{D}^{\mathrm{NR}}(s)} .
\end{aligned}
$$

As discussed in Sec. II C of Part I, the GGG instrument must be as sensitive as possible to low frequency effects (between $10^{-5}$ and $10^{-4} \mathrm{~Hz}$ ). For this reason, in the following we shall focus on the $\chi(s \rightarrow 0) \equiv \chi_{0}$ behavior of the rejection factor for different values of the spin frequency $\nu_{s}$ of the GGG rotor.

\section{B. Nonspinning rotor: Analytical solution and scaling parameter}

We first compute the rejection factor in the particular case of zero spin rate, i.e., for the nonspinning GGG apparatus, showing that the capability of the system to reject common mode forces can be predicted analytically, and that rejection is quantitatively expressed by a simple scale parameter. The model we use to describe the GGG apparatus is the same as in Fig. 3 of Part. I

The relative displacement $\Delta x_{D}$ of the test cylinders in response to an external acceleration $a_{D}$, acting in differential mode, can be written as

$$
\Delta x_{D}=\frac{a_{D} T_{D}^{2}}{(2 \pi)^{2}}=\frac{a_{D} m_{t} L_{a}^{2}}{K_{t} l^{2}-g m_{t} \Delta L / 2},
$$

where the second equality is obtained by using the expression for the natural period of differential oscillation of the test cylinders $T_{D}$ as computed in Part I, Eq. (41), namely,

$$
T_{D}=\frac{2 \pi}{\sqrt{\left[\left(K+K_{i}+K_{o}\right) l^{2} /\left(m_{i}+m_{o}\right) L_{a}^{2}\right]-\left(g / 2 L_{a}\right)\left(\Delta L / L_{a}\right)}},
$$

and introducing the total mass of the test bodies $m_{t}=m_{i}$ $+m_{o}$ and the total elastic constant $K_{t}=K+K_{i}+K_{o}$ (assuming isotropic suspensions).

Let us now see how an external acceleration $a_{C}$, albeit applied in common mode (i.e., the same on both test cylinders), will nevertheless affect their relative position giving rise to a relative displacement $\Delta x_{C}$. Note that the system is at equilibrium, it is not rotating, and we are limiting the calculation to small angles and to constant applied forces (i.e., to forces which are dc in the nonrotating laboratory frame). We then have

$$
\Delta x_{C}=L_{o} \theta_{o}-L_{i} \theta_{i}-\left(2 L_{a}+\Delta L\right) \theta_{a} .
$$

We now need the values of $\theta_{\lambda}$ in the presence of a common mode force (the label $\lambda=i, o, a$ refers to the inner mass, outer mass, and coupling arm, respectively, as in Part I). They can be obtained from the equation

$$
\left.\frac{\partial U}{\partial q_{j}}\right|_{q_{j}=q_{j}^{0}}=0, \quad j=1, \ldots, n,
$$

[already given as Eq. (22) in Part I] in the limit of small angles, having added to the potential energy $U$, the work done by the external forces. After some algebra, this procedure leads to the following equations:

$$
\begin{aligned}
& m_{i} g L_{i} \theta_{i}-K_{i} l^{2}\left(\theta_{a}-\theta_{i}\right)-m_{i} a_{C} L_{i}=0, \\
& m_{o} g L_{o} \theta_{o}-K_{o} l^{2}\left(\theta_{a}-\theta_{o}\right)-m_{o} a_{C} L_{o}=0, \\
& {\left[K_{t} l^{2}-\frac{1}{2}\left(m_{t}+m_{a}\right) g \Delta L\right] \theta_{a}-K_{i} l^{2} \theta_{i}-K_{o} l^{2} \theta_{o}+\frac{1}{2}\left(m_{t}\right.} \\
& \left.\quad+m_{a}\right) a_{C} \Delta L=0 .
\end{aligned}
$$

After some additional manipulations, Eqs. (12) yield the approximated values of the angles as

$$
\begin{aligned}
\theta_{i} & \simeq \frac{m L_{i}}{K_{i} l^{2}+m g L_{i}} a_{C}, \\
\theta_{o} & \simeq \frac{m L_{o}}{K_{o} l^{2}+m g L_{o}} a_{C}, \\
\theta_{a} & \simeq-\frac{(1 / 2) m_{t} \Delta L-\sum_{\lambda=i, o} K_{\lambda} l^{2}\left(m L_{\lambda} / K_{\lambda} l^{2}+m g L_{\lambda}\right)}{K_{t} l^{2}-(1 / 2) m_{t} g \Delta L} a_{C} .
\end{aligned}
$$

After expanding Eqs. (13) in the small parameters $K_{\lambda} l^{2} / m_{\lambda} g L_{\lambda}$ and substituting the resulting equations into (10) using the relation $L_{o}=2 L_{a}+\Delta L+L_{i}$, we eventually obtain

$$
\Delta x_{C} \simeq \frac{\left(2 L_{a}+\Delta L\right) K l^{2}}{\left[K_{t} l^{2}-(1 / 2) m_{t} g \Delta L\right] g} a_{C} .
$$

The ratio of the relative displacement $\Delta X_{D}$ caused by a differential force, over the relative displacement $\Delta X_{C}$ caused by a common mode force (along the $X^{\prime}$ direction of the non rotating frame), is therefore

$$
\frac{\Delta X_{D}}{\Delta X_{C}}=\frac{m_{t} g L_{a}^{2}}{\left(2 L_{a}+\Delta L\right) K l^{2}} \frac{a_{D}}{a_{C}},
$$

which, for $a_{D}=a_{C}$, gives us the inverse of the rejection factor along the same direction of the horizontal plane,

$$
\frac{1}{\chi_{0}}=\frac{m_{t} g L_{a}^{2}}{\left(2 L_{a}+\Delta L\right) K l^{2}},
$$

that is, an external acceleration acting on the GGG test cylinders in common mode would produce a relative displacement of the cylinders with respect to one another $1 / \chi_{0}$ times smaller than the same acceleration would produce if acting in differential mode. For a perfectly differential instrument, $1 / \chi_{0}$ would be infinite, namely, a common mode force would not produce any relative displacement of the test masses. Here we indicate the rejection factor with the subscript zero because this analytical calculation refers to the rejection of dc external forces (i.e., of forces which act at zero frequency in the laboratory frame). In the following numerical computation we will also show the dependence of the rejection factor on the frequency of the applied force, as well as on the rotation speed of the GGG rotor.

The rejection factor (16) takes a very simple form in the limits $\Delta L / L_{a} \rightarrow 0$ and $m_{a} / m_{i, o} \rightarrow 0$, that are verified in our experiment. This is 


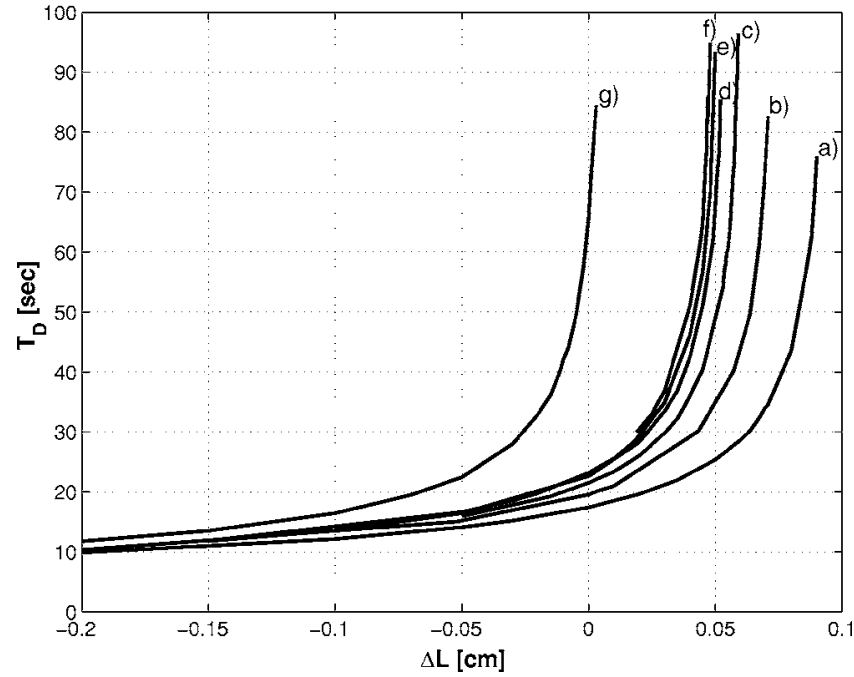

FIG. 1. Differential period $T_{D}$ as a function of the balancing parameter $\Delta L$. The various curves refer to different values of the other parameters of the system, as given in Table I (all simulations were performed with the rotor spinning at $\nu_{s}=2.5 \mathrm{~Hz}$ ).

$$
\frac{1}{\chi_{0}} \simeq \frac{m_{i, o} g L_{a}}{K l^{2}} .
$$

Thus, at zero spin, the inverse of the common mode rejection factor $1 / \chi_{0}$ is given by the simple scaling parameter (17), where the relevant energy scales are the gravitational energy of the inner and outer test cylinders (at the numerator) and the elastic energy stored by the central suspension (at the denominator). The larger is this ratio, the better the instrument will reject common mode forces, the more suitable it will be to detect differential effects such as that of an equivalence principle violation. In the following we show that, far from being limited to the very particular case of zero spin rate, this result holds also for the spinning rotor in the region of low and high spin frequencies, as defined in Sec. II A.

\section{Region of low and high spin frequencies}

Expression (17), for the rejection factor of dc common mode forces, results from a number of approximations performed in describing the system in the case of zero spin rate. We devote this section to evaluate numerically to which extent it is valid also in the low and high spin frequency regions (see Sec. II A). The more complex case of the rejection behavior, when the rotor is spinning at intermediate frequencies, will be addressed in Sec. III D.

\section{The differential period $T_{D}$}

In order to calculate the dependence of the rejection on the scaling parameter $K l^{2} / m_{i, o} g L_{a}$, we proceed by varying one at a time its governing parameters. We do that while keeping the differential period $T_{D}$ fixed, by also varying $\Delta L$ (see Fig. 1, where $T_{D}$ vs $\Delta L$ is displayed under the different experimental conditions listed in Table I). $T_{D}$ must be kept fixed because its variation would mean a variation of the stiffness of the coupling between the test cylinders, and therefore a different response, in terms of relative displacement, under the action of a given external force. The softer
TABLE I. Legend corresponding to Fig. 1.

\begin{tabular}{lccc}
\hline \hline Curve & $\begin{array}{l}K_{X^{\prime}} \\
(\mathrm{dyn} / \mathrm{cm})\end{array}$ & $\begin{array}{c}\Lambda \\
\left(K_{Y^{\prime}} / K_{X^{\prime}}\right)\end{array}$ & $\begin{array}{c}l \\
(\mathrm{~cm})\end{array}$ \\
\hline $\mathrm{a}$ & $10^{6}$ & 2.58 & 0.5 \\
$\mathrm{~b}$ & $10^{6}$ & 1 & 0.5 \\
$\mathrm{c}$ & $5 \times 10^{5}$ & 1 & 0.5 \\
$\mathrm{~d}$ & $2.5 \times 10^{5}$ & 1 & 0.5 \\
$\mathrm{e}$ & $1.5 \times 10^{5}$ & 1 & 0.5 \\
$\mathrm{f}$ & $5 \times 10^{4}$ & 1 & 0.5 \\
$\mathrm{~g}$ & $10^{6}$ & 1 & 0.15 \\
\hline \hline
\end{tabular}

the coupling, the longer the differential period, the larger the relative displacement between the test cylinders in response to a given force.

\section{Spectra of the test mass differential displacements}

Once the differential period is fixed, we need to set the observables that are needed in order to extract the rejection factor. We first need to establish how the signal of the relative displacements of the test cylinders (in the nonrotating frame) responds to the frequency of the external force applied, either in common mode or in differential mode [see Eqs. (4) and (5)], for a given spin frequency $\nu_{s}$ of the rotor.

We evaluate numerically Eqs. (4) and (5). Figure 2 shows the magnitude of the relative displacement resulting from the application, along the $X^{\prime}$ direction of the nonrotating frame, of a common mode acceleration (top panel) and of a differential one (bottom panel), of the same intensity, varying at a frequency that ranges between $10^{-5}$ and $10 \mathrm{~Hz}$, with the rotor spinning at frequency $\nu_{s}=2.5 \mathrm{~Hz}$. Though the force is applied in the $X^{\prime}$ direction, there will be some effect also in the perpendicular $Y^{\prime}$ direction, as discussed below in relation to Fig. 3. Here we show only the effect in the direction $X^{\prime}$ of the force.

In the case of common mode input accelerations (Fig. 2, top panel), the test masses of the rotor are seen to respond with a relative displacement at all the natural frequencies. The plot shows peaks at the frequencies $\nu_{\text {pole }}$ corresponding to the differential frequency $\nu_{D} \simeq 0.09 \mathrm{~Hz}$, to the common ones $\nu_{C 1} \simeq 0.9 \mathrm{~Hz}$ and $\nu_{C 2} \simeq 1.26 \mathrm{~Hz}$, and to their combinations with $2 \nu_{s}$, namely, $2 \nu_{s} \pm \nu_{D}, 2 \nu_{s} \pm \nu_{C 1}$, and $2 \nu_{s} \pm \nu_{C 2}$. Two zeros of the transfer function are also apparent, the first located in between $\nu_{D}$ and $\nu_{C 1}$ and the second in between $\nu_{C 1}$ and $\nu_{C 2}$.

In the case of differential input accelerations (Fig. 2, bottom panel), no zeros are present in the transfer function, and only the mode at frequency $\nu_{D}$ is significantly excited, while the effect at $2 \nu_{s} \pm \nu_{D}$ is negligible. The value of the relative displacement for $\nu \rightarrow 0$ (i.e., as the applied force becomes almost dc) turns out to be in perfect agreement with the value predicted by Eq. (8) for the zero spin case, though this figure refers to the system spinning at $\nu_{s}=2.5 \mathrm{~Hz}$.

The corresponding inverse rejection factor, as given by Eqs. (6) and (7) for the two directions of the horizontal plane, is displayed in Fig. 3. Even though the external forces (both common and differential) have been applied along the $X^{\prime}$ direction only, differential displacements occur also along $Y^{\prime}$, because of losses in the test mass suspensions while ro- 

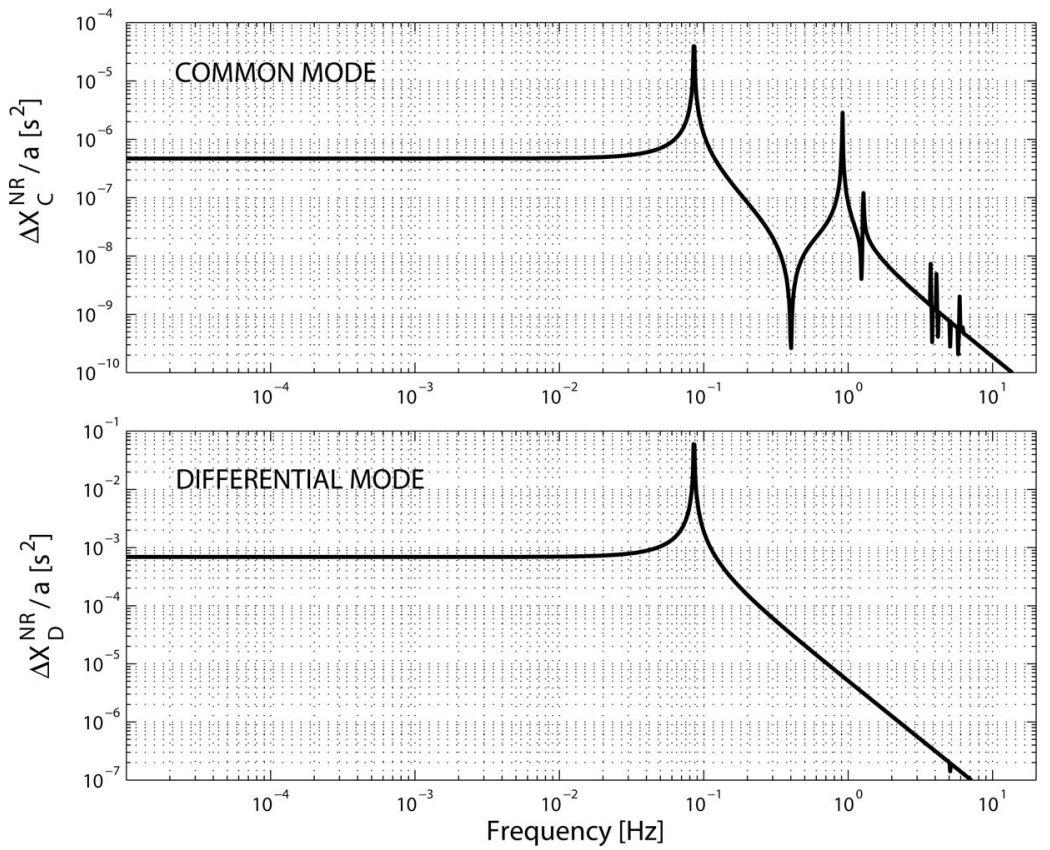

FIG. 2. Common mode $\Delta x_{C}^{\mathrm{NR}}$ (top panel) and differential mode $\Delta x_{D}^{\mathrm{NR}}$ (bottom panel) relative displacements, divided by the intensity $a$ of the acceleration applied, in common mode or differential mode, respectively, as functions of the frequency of the applied force. The rotor is spinning at $\nu_{s}=2.5 \mathrm{~Hz}$. The other parameters of the system are typical of the present instrument: $T_{D}$ $=12.5 \mathrm{~s}, \quad K=K_{i}=K_{o}=10^{6} \mathrm{dyn} / \mathrm{cm}, \quad l=0.5 \mathrm{~cm}, \quad L_{a}$ $=19 \mathrm{~cm}, m_{i, o}=10 \mathrm{~kg}$, and $L_{i}=4.5 \mathrm{~cm}$. tating (as discussed in Part $\mathrm{I}$, the quality factor $Q$ is finite in our simulations). For this reason, the spectrum along the $Y^{\prime}$ direction shows an additional peak at the differential mode frequency. However, the magnitudes of both the common and differential $Y^{\prime}$ displacements are very small, reduced by a factor $Q$ with respect to those along $X^{\prime}$ (their ratio remaining of the same order of magnitude as that in the $X^{\prime}$ direction).

\section{Rejection of dc forces versus the governing parameters}

We now vary-one at a time-all the four governing parameters which appear in the scaling parameter (17), plus the anisotropy factor $\Lambda$ of the suspensions introduced in Part I, Sec. IV. If $\Lambda=1$ the suspensions have the same stiffness in both directions of the horizontal plane; if not, there is an anisotropy (see Table I). The purpose is to determine how the rejection factor of dc common mode forces, $\left|1 / \chi_{0}\right|$, depends on these parameters both at zero and high spin frequencies. In doing this we need to keep the natural differential period $T_{D}$ fixed, as discussed above. Figure 4 displays, in its five panels, the dependence of $\left|1 / \chi_{0}\right|$ on the five relevant parameters (the balancing arm length $L_{a}$, the mass $m_{i, o}$ of the suspended cylinders, the elastic constant $K$ of the central laminar suspension, its length $l$, and the anisotropy factor $\Lambda$ ). In each panel, the solid lines give the value of $\left|1 / \chi_{0}\right|$ for the zero spin case, while the filled circles give its value for the rotor spinning at $2.5 \mathrm{~Hz}$. We are therefore investigating the rejection of $\mathrm{dc}$ forces in what we call the very low and very high spin frequency regions of the rotor.

As all five panels in Fig. 4 show, there is almost no difference between the zero spin and $2.5 \mathrm{~Hz}$ spin frequency cases. This result had to be expected from our analysis of the normal modes of the GGG system developed in Part I (and reported in Fig. 4 therein), where it was apparent that the horizontal branches and the inclined ones (at $2 \nu_{s}$ ) of the normal modes do not cross in the low and high spin frequency regions. By performing a spectral analysis of the experimental data we have verified that the horizontal branches of the normal modes are typically excited, while the inclined ones are not. Thus, if no crossing occurs, also no energy transfer occurs from the former to the latter.

We expect this not to be the case in the intermediate spin frequency region, where the horizontal and the inclined branches of the normal modes do cross (see the analysis of this region in Sec. III D below).

\section{Validation of the scaling parameter}

We can now collect all the results discussed so far in order to quantify the validity of the scaling parameter (17) in determining the rejection of dc common mode forces. The results of our numerical simulations are reported in Fig. 5, where we plot $\left|1 / \chi_{0}\right|$ as a function of the scaling parameter
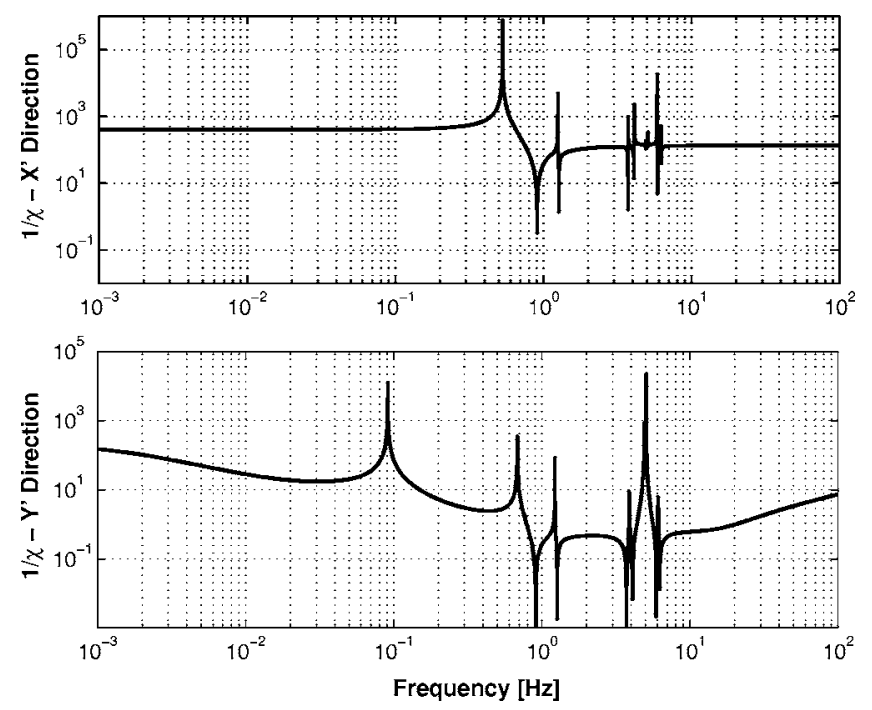

FIG. 3. Inverse rejection function $1 / \chi(\nu)$ vs frequency in the $X^{\prime}$ (top) and $Y^{\prime}$ (bottom) directions for the rotor spinning at $\nu_{s}=2.5 \mathrm{~Hz}$. The other system parameters are the same as in Fig. 2. 


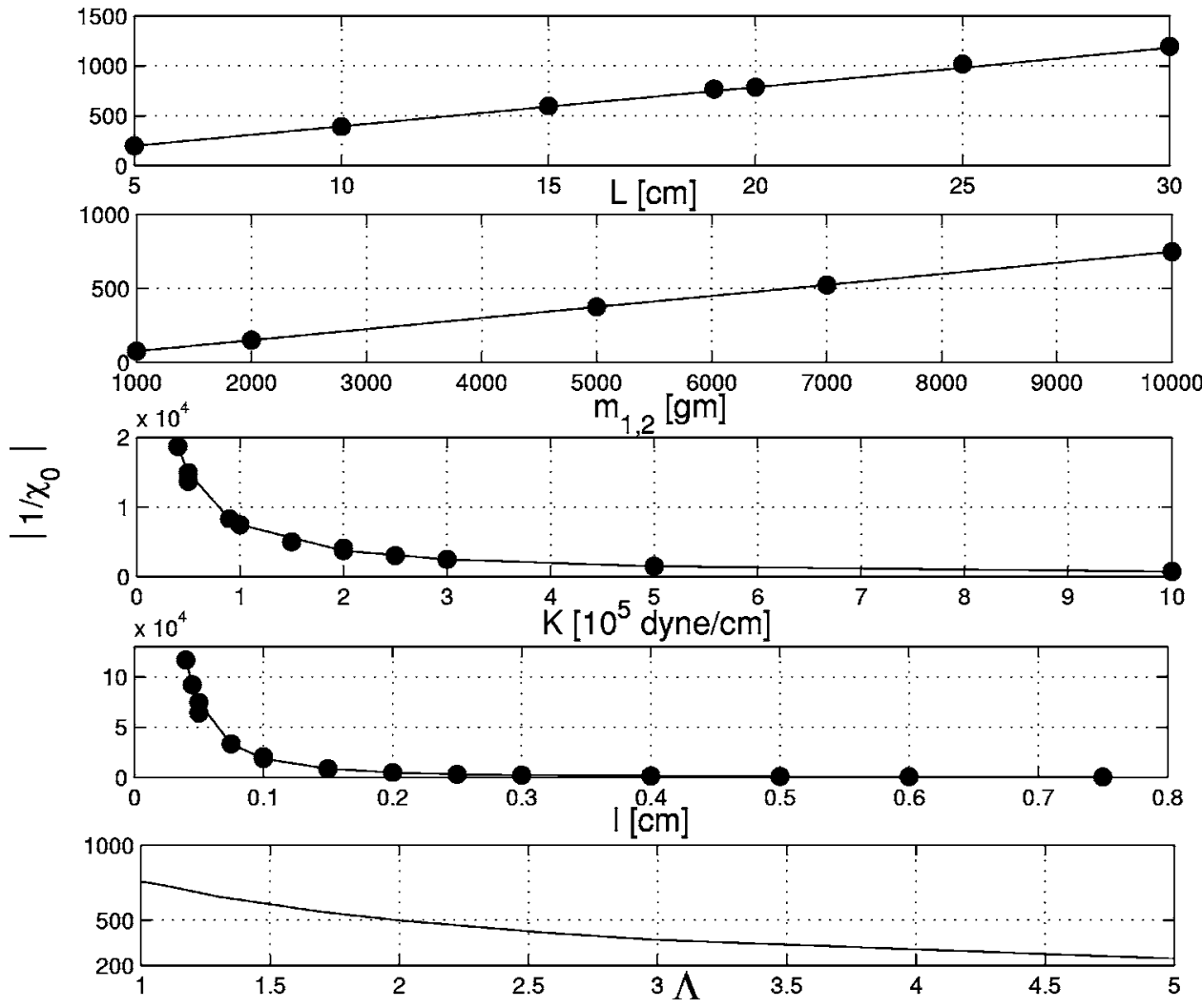

FIG. 4. Inverse rejection factor of dc forces, $1 / \chi_{0}$, as a function of various system parameters. From top to bottom, the varying parameters are $L_{a}$, $m_{i, o}, K, l$, and the anisotropy $\Lambda$. Solid line: nonspinning rotor. Points: rotor spinning at $\nu_{s}=2.5 \mathrm{~Hz}$. The parameters are changed one at a time from the values reported in the caption of Fig. 2.
(17). The solid line at $45^{\circ}$ represents $\left|1 / \chi_{0}\right|$ in the case of a nonspinning rotor with isotropic suspensions $(\Lambda=1)$, and it has been found to be valid also for the isotropic spinning rotor in the low and high spin frequency regions. If then anisotropy is taken into account, the resulting values of $\left|1 / \chi_{0}\right|$ still lie on the $45^{\circ}$ line as long as the spin frequency is

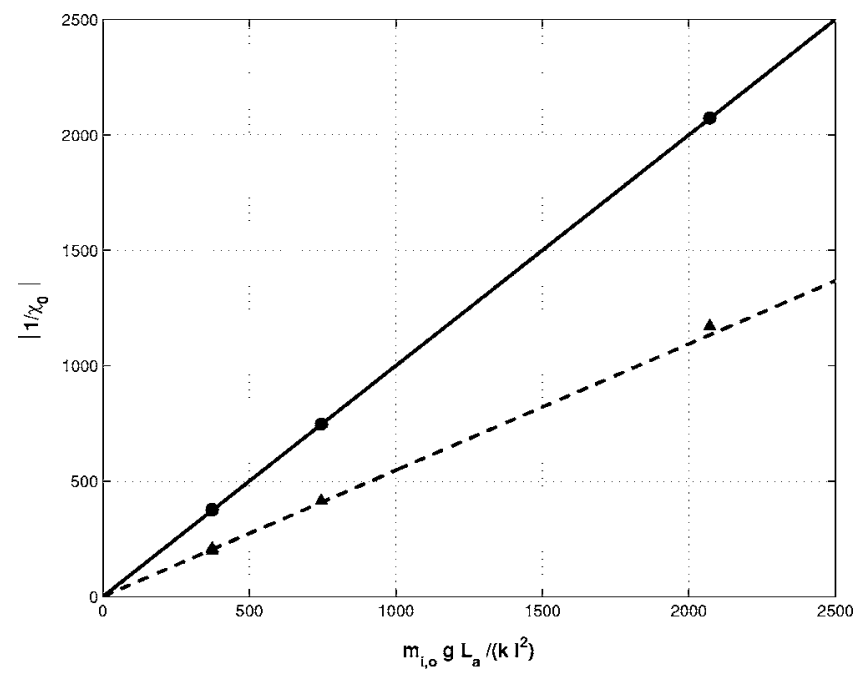

FIG. 5. Results from numerical simulations of the inverse rejection factor of $\mathrm{dc}$ forces, $1 / \chi_{0}$, as a function of the scaling parameter $m_{i, o} g L_{a} /\left(K l^{2}\right)$. The solid line refers to the zero spin case with isotropic suspensions $(\Lambda=1)$, and also to the isotropic rotor in the low and high spin frequency regions. Once anisotropy of the suspensions is taken into account (e.g., with $\Lambda=2.58$ ), the rotor spinning at low frequencies gives the results shown as filled circles, while the one spinning at high frequencies gives the results shown as filled triangles. The dashed line has no physical meaning; it simply shows that the filled triangles still lie on a line, though at lower inclination. The system parameters reported in Fig. 2 correspond to $m_{i, o} g L_{a} /\left(K l^{2}\right)=745$. very low (filled circles), while they lie on a lower inclination line if the spin frequency is very high (filled triangles). That is, in the latter case, the inverse rejection factor of dc common mode forces $\left|1 / \chi_{0}\right|$ is still proportional to the scaling parameter (17), but through a coefficient smaller than unity. The amount of the deviation depends on $\Lambda$, as shown in the bottom panel of Fig. 4.

\section{Region of intermediate spin frequencies}

We now compute the inverse rejection factor $1 / \chi(\nu)$ for a wide range of frequency $\nu$ of the applied force, in the region of intermediate values of the spin frequency of the rotor (as defined in Sec. II A). The calculations are similar to those which led to Fig. 3 in the case of high spin frequency $(2.5 \mathrm{~Hz}$ in that case). Since we are interested in applied forces of very low frequency, only the value $\left|1 / \chi_{0}\right|$ of the inverse rejection factor for a dc applied force is plotted as a function of the spin frequency $\nu_{s}$ (Fig. 6, top panel). This figure shows very clearly that the best performance of the instrument (i.e., best rejection of common mode dc forces) is to be expected, with the current parameters of the instrument, at the values $\nu_{s} \simeq 0.36 \mathrm{~Hz}>\nu_{D}$ and $\nu_{s} \simeq 0.6 \mathrm{~Hz}>\nu_{D}$, where the value of $1 / \chi_{0}$ is as high as $10^{6}$. The difference between the values of $\left|1 / \chi_{0}\right|$ at the two ends of the spin frequency (for $\nu_{s} \rightarrow 0$ and $\left.\nu_{s} \rightarrow \infty\right)$ is due to the anisotropy of the central suspension, as already shown in Fig. 5. We have run the same system as in Fig. 6, but with isotropic elastic constants, and have numerically verified that $\left|1 / \chi_{0}\left(\nu_{s}=0\right)\right|=\mid 1 / \chi_{0}\left(\nu_{s}\right.$ $=\infty) \mid$, the positions of the peaks being slightly changed according to the corresponding change in the differential period. 

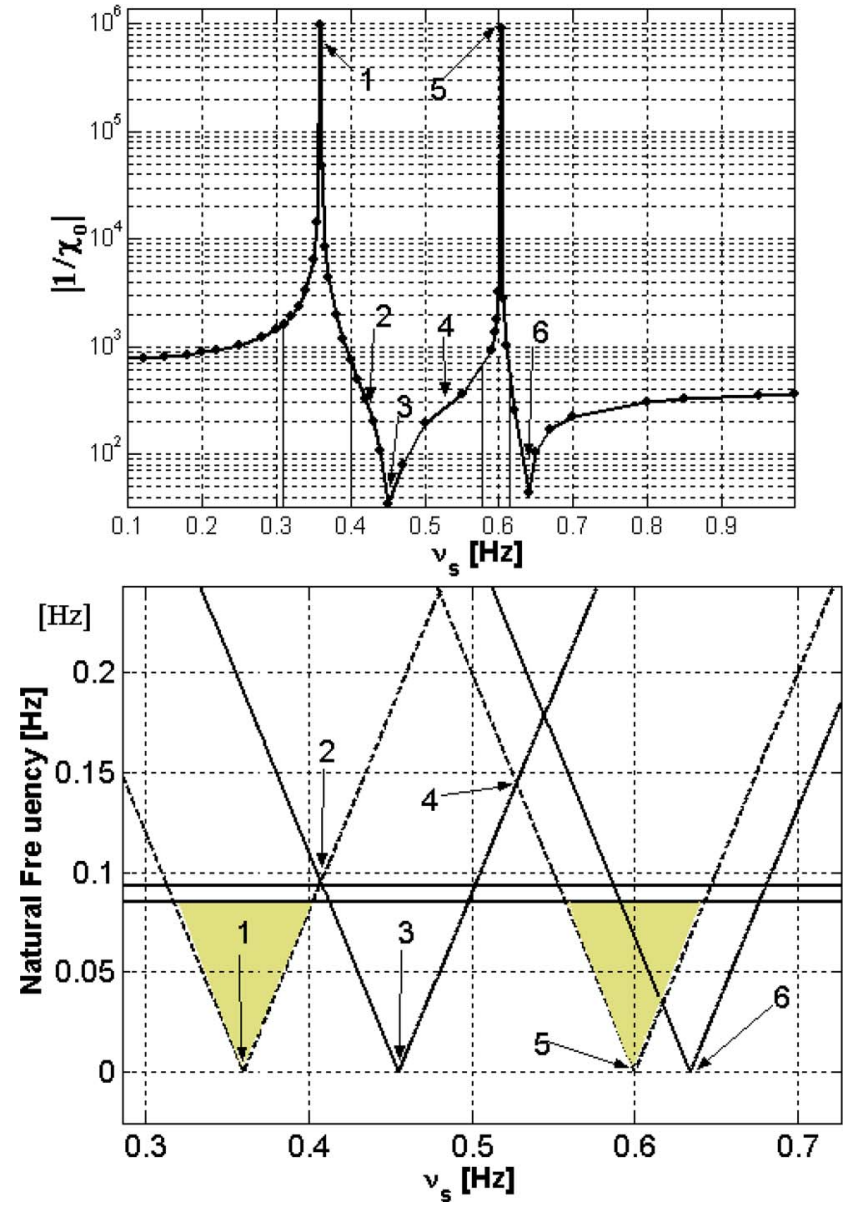

FIG. 6. Top panel: the inverse rejection factor of common mode dc forces, $1 / \chi_{0}$, as a function of the spin frequency $\nu_{s}$. The system parameters are the same as in Fig. 2. The numbered arrows indicate crossing points and minima (see text) and correspond to those shown in the bottom panel. Bottom panel: absolute values of the zeros (dashed lines) and of the poles (solid lines) of the transfer function vs $\nu_{s}$. The horizontal branches correspond to the differential frequencies $\nu_{D}$, and split because of the anisotropy. For $\nu_{s}$ within the shaded areas, the response is dominated by the zeros of the transfer function $H(s)$, and therefore the relative displacement in response to common mode de forces, $\Delta x_{C}(\nu \rightarrow 0)$, is strongly suppressed.

The enhanced rejection behavior at intermediate spin frequencies is related to the dependence of the zeros and of the poles of the transfer matrix on $\nu_{s}$, as we are going to discuss below. As it happens in the case of the poles of the transfer function, also the values of its zeros change with the spin frequency, showing the typical two branch behaviors, namely, a flat branch and an inclined one with $2 \nu_{s}$ coefficient. This is apparent in the bottom panel of Fig. 6, where we plot the absolute values of the zeros and of the poles of the transfer function, $\left|\nu_{\text {zero }}\right|$ and $\left|\nu_{\text {pole }}\right|$, as dashed and solid lines, respectively.

The nondispersive branches in Fig. 6, bottom panel, shown as solid horizontal lines, correspond to the poles at the differential frequencies $\nu_{D}$ (there are two of them because of the anisotropy in the suspensions). The zero branches, represented by dashed lines in the same figure, are characterized by minima located at $0.5\left|\nu_{\text {zero }}\left(\nu_{s}=0\right)\right|$ and marked with the numbers 1 and 5. The zero minima are shifted from the minima of the pole branch, that are located at the points marked as 3 and 6 . At the points marked as 2 and 4 , the zero branch with $-\left|\nu_{\text {zero }}\right|+2 \nu_{s}$ crosses the pole branch with $-\left|\nu_{\text {pole }}\right|+2 \nu_{s}$.

We thus see that there are ranges of spin frequencies for which $\left|\nu_{\text {zero }}\right|<\nu_{D}$. This occurs within the shaded region of the frequencies indicated in the bottom panel of Fig. 6, whose width is easily evaluated to be precisely $\nu_{D}$. When $\left|\nu_{\text {zero }}\right|<\nu_{D}$, the low frequency rotor response is dominated by the position of the zero, hence the value of the relative displacement $\Delta x_{C}(\nu \rightarrow 0)$ of the test cylinders in response to a low frequency common mode force is strongly suppressed (i.e., the disturbance is strongly rejected).

In order to make the correspondence clear, we have reported in the top panel of Fig. 6 the same points 1-5 marked in the bottom one. We thus see that the peaks of $\left|1 / \chi_{0}\right|$ correspond to the minima 1 and 5 of the zero branches, the valleys of $\left|1 / \chi_{0}\right|$ correspond to the minima 3 and 6 of the pole branches, and finally the saddle points of $\left|1 / \chi_{0}\right|$ correspond to the crossings 2 and 4 between zero and pole branches.

The fundamental question then arises as to how we can move the location of the peaks shown in the top panel of Fig. 6 in order to enhance the capability of the instrument to reject common mode forces at larger supercritical values of the spin frequency $\nu_{s}$, since rotation provides signal modulation and higher frequency modulation is preferable. We are going to address this question in the next section.

\section{ENHANCED REJECTION BEHAVIOR OF THE GGG ROTOR}

In Sec. III C we have shown that in the region of low and high spin frequencies the scaling parameter $m_{i, o} g L_{a} /\left(K l^{2}\right)$ precisely describes the rejection behavior of the GGG rotor, the differential period being adjusted for every set of parameters by varying $\Delta L$. Then, in Sec. III D we have investigated the region of intermediate spin frequencies showing how the spin frequency can be tuned so as to obtain a considerably enhanced rejection of common mode forces in a nontrivial manner. We now need one more independent "knob" in order to move the $1 / \chi_{0}$ peaks towards higher spin frequencies, where we expect a better performance of the GGG experiment.

If we vary the scaling parameter in the region of intermediate spin frequencies, the results obtained are shown in Fig. 7: as the value of the scaling parameter increases, the inverse rejection factor somewhat improves at the two ends of the plot, but the position of the peaks, namely, the spin frequencies at which rejection is strongly enhanced, is unaffected.

However, we can still vary the remaining free parameters $L_{i, o}$ while keeping $m_{i, o} g L_{a} /\left(K l^{2}\right)$ fixed. Figure 8 shows that, as the values of $L_{i}$ (Ref. 12) increase in going from the bottom to the top panel of the figure, the separation in spin frequency between the peaks of $1 / \chi_{0}$ increases too.

All the cases displayed in Fig. 8 refer to a realistic GGG apparatus. In particular, in all three panels the scaling parameter has always the same value $m_{i, o} g L_{a} /\left(K l^{2}\right)=370$ (with $K$ $=10^{5} \mathrm{dyn} / \mathrm{cm}, L_{a}=19 \mathrm{~cm}, l=1 \mathrm{~cm}$, and $m_{i, o}=10 \mathrm{~kg}$ ). Only the value of $L_{i}$ increases from 4.5 to 9.0 to $15 \mathrm{~cm}$ in going 


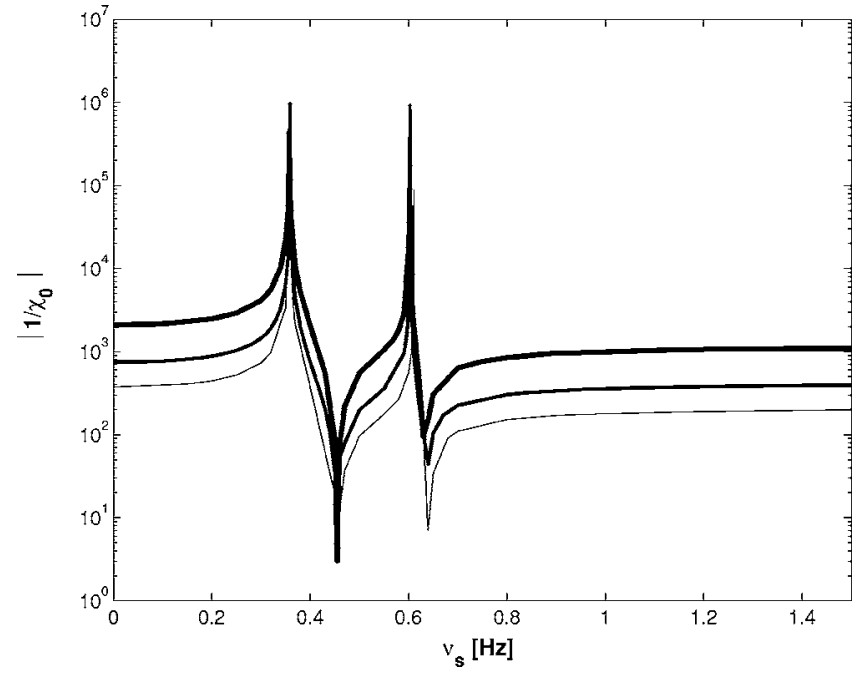

FIG. 7. Inverse static rejection $1 / \chi_{0}$ as a function of the spin frequency. Curves of increasing thickness refer to increasing values of the scaling parameter $m_{i, o} g L_{a} /\left(K l^{2}\right)=370,745$, and 2070 , while keeping $L_{i}=4.5 \mathrm{~cm}$ fixed. We note that $1 / \chi\left(\nu_{s} \rightarrow 0\right) \neq 1 / \chi\left(\nu_{s} \rightarrow \infty\right)$ because of the anisotropic central suspension. Note that different values of $m_{i, o} g L_{a} /\left(K l^{2}\right)$ leave the position of the peaks unaffected.

from the bottom to the top panel. At the top panel, with $L_{i}$ $=15 \mathrm{~cm}$, the inverse rejection factor of common mode $\mathrm{dc}$ forces has a peak as high as $1 / \chi_{0}=1.5 \times 10^{5}$ at a spin frequency $\nu_{s}=1.12 \mathrm{~Hz}$.

Figure 8 summarizes the main result of this work, as it shows the way to perform a controlled tailoring of the rejection capability of the GGG apparatus. This can be done essentially by tuning $L_{i}$ and $\nu_{s}$. In the experiment, the most convenient way is to first fix $m_{i, o} g L_{a} /\left(K l^{2}\right)$ and $L_{i}$ in such a way that $\left|\nu_{\text {zero }}\left(\nu_{s}=0\right)\right| \simeq 2 \nu_{s}^{\max }$, where $\nu_{s}^{\max }$ is the spin frequency at which the rotor is finally operated (and at which we want the best performance). A finer tuning is then done
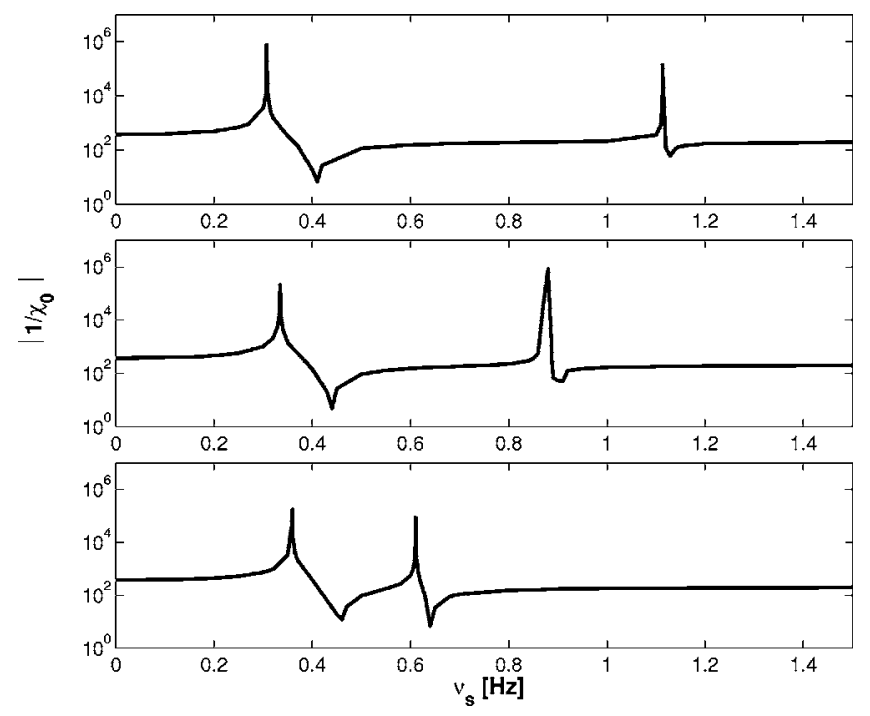

FIG. 8. Inverse rejection factor of common mode dc forces, $1 / \chi_{0}$, as a function of the spin frequency at different values of $L_{i}$ [with the scaling parameter fixed at $\left.m_{i, o} g L_{a} /\left(K l^{2}\right)=370\right]$. From bottom to top: $L_{i}=4.5 \mathrm{~cm}$, $L_{i}=9.0 \mathrm{~cm}$, and $L_{i}=15.0 \mathrm{~cm}$. Note the increasing separation in frequency between the peaks from bottom to top, leading to enhanced rejection at higher spin frequencies. For the maximum separation case (top panel) enhanced rejection takes place at $\nu_{s}=1.12 \mathrm{~Hz}$. by adjusting $\nu_{S}$ so as to bring the first zero below the differential frequency $\nu_{D}$, as shown in the bottom panel of Fig. 6. This can be done in a highly controlled way, allowing us to place the system in correspondence to the peaks of $\left|1 / \chi_{0}\right|$.

\section{CONCLUDING REMARKS AND PERSPECTIVES}

We have investigated the frequency-dependent response of the GGG rotating differential accelerometer for testing the equivalence principle using an effective physical model (Part I, Sec. III) along with a simulation procedure (Part I, Sec. IV and Part II, Sec. II). This method has been demonstrated to quantitatively account for the available experimental data (Part I, Sec. II) and to provide analytical insights helpful for a qualitative understanding of the underlying physics.

In Part I we have shown, among other things, the split up of the normal modes into two scissor like branches, distinguishing modes which are preferentially excited from those whose spectral amplitudes are typically small, thus learning how to avoid the spin frequencies corresponding to their crossings, in order not to excite the quiet modes too by exchange of energy. We have also investigated the selfcentering characteristic of the GGG rotor when in supercritical rotation regime, gaining insight on how to exploit this very important physical property for improving the quality of the rotor, hence its sensitivity as differential accelerometer. Here we can add a major result. The rejection of common mode dc forces is characterized by two distinct behaviors, depending on the region of spin frequency $\nu_{s}$ at which the rotor is operated. For low and high values of $\nu_{s}$, the dependence of the inverse rejection factor $1 / \chi_{0}$ is quantitatively expressed by the scaling factor $m_{i, o} g L_{a} /\left(K l^{2}\right)$, with all the relevant parameters combined in it. In the case of intermediate values of $\nu_{s}, 1 / \chi_{0}$ can reach peaks as high as $10^{5}-10^{6}$, whose positions are affected by the remaining parameters $L_{i, o}$ and $K_{i, o}$. This conclusion allows us to tailor the features of the real instrument for best performance in terms of rejection of external disturbances such as tidal forces and seismic noise. Future experimental tests can probe this conclusion.

Results from both Parts I and II indicate that we can aim at a more realistic simulation model, to be used online with the experiment, as the latter becomes more sensitive (e.g., by reduction of the motor disturbances, by remote adjustment of the verticality of the spin axis, by active control of low frequency terrain tilt noise, etc.). The key point is that changes can be easily implemented in our numerical method and simulation environment, since any modification of the model corresponds to modifying only the part of the code where the Lagrange function is clearly written in terms of vector operations. The possibility to perform such realistic simulations, before applying any real changes to the apparatus, allows us to implement those which provide the best results so as to optimize the experiment. Along these lines, the present approach can be adapted to the "Galileo Galilei" (GG) experiment in space, where we expect a much more sensitive test of the equivalence principle. 


\section{ACKNOWLEDGMENT}

Thanks are due to INFN for funding the GGG experiment in its laboratory of San Piero a Grado in Pisa.

\section{APPENDIX: THE TRANSFER FUNCTION}

\section{The $C$ matrix}

In our experiment, Eq. (3) is characterized by two inputs, namely, the $X^{\prime}$ and $Y^{\prime}$ components of the external forces, and two outputs $Y$, that are the relative displacements of the two test cylinders from their equilibrium positions in the sensitivity plane as measured in the rotating $X^{\prime} Y^{\prime}$ frame. That is, $Y \equiv\left\{Y_{1}, Y_{2}\right\}=\left[\Delta \mathbf{R}_{o}(t)-\Delta \mathbf{R}_{i}(t)\right] \cdot\left\{\hat{X}^{\prime}, \hat{Y}^{\prime}\right\}, \quad$ where $\Delta \mathbf{R}_{\lambda}(t)=\mathbf{R}_{\lambda}(t)-\mathbf{R}_{\lambda}^{0}$. In order to determine the coefficients of the $C$ matrix, we thus have to combine Eq. (2) of Part I for the vectors pointing to the three bodies and written in terms of the unit vectors $\hat{L}_{a}, \hat{L}_{o}$, and $\hat{L}_{i}$ together with the expression (16) of Part I for the unit vectors in terms of the generalized coordinates $X=\left\{x_{1}, \ldots, x_{12}\right\}$, thereby obtaining expressions for $\mathbf{R}_{\lambda}\left(\left\{x_{1}, \ldots, x_{12}\right\}\right)$. In the linearized theory, we then have $\Delta \mathbf{R}_{\lambda}(t)=\mathbf{R}_{\lambda}\left(\left\{x_{1}+x_{1}^{0}, \ldots, x_{12}+x_{12}^{0}\right\}\right)-\mathbf{R}\left(x_{1}^{0}, \ldots, x_{12}^{0}\right)_{\lambda}$, so that we can explicitly form the differences

$$
\begin{aligned}
& Y_{1}=\hat{X}^{\prime} \cdot\left[\mathbf{R}_{o}\left(X+X^{0}\right)-\mathbf{R}_{o}\left(X^{0}\right)-\mathbf{R}_{i}\left(X+X^{0}\right)+\mathbf{R}_{i}\left(X^{0}\right)\right], \\
& Y_{2}=\hat{Y}^{\prime} \cdot\left[\mathbf{R}_{o}\left(X+X^{0}\right)-\mathbf{R}_{o}\left(X^{0}\right)-\mathbf{R}_{i}\left(X+X^{0}\right)+\mathbf{R}_{i}\left(X^{0}\right)\right] .
\end{aligned}
$$

After imposing

$$
\left(\begin{array}{l}
Y_{1} \\
Y_{2}
\end{array}\right)=C\left(\begin{array}{c}
x_{1} \\
x_{2} \\
\cdots \\
x_{12}
\end{array}\right),
$$

we obtain the coefficients $C_{j k}$ of the $2 \times 2 n$ matrix as

$$
\begin{aligned}
& C_{1 k}=\gamma_{k} \cos x_{k}^{0} \cos x_{k+6}^{0}, \quad k \leqslant 6, \\
& C_{1 k}=\gamma_{k} \sin x_{k-6}^{0} \sin x_{k}^{0}, \quad k>6, \\
& C_{2 k}=\gamma_{k} \cos x_{k}^{0} \sin x_{k+6}^{0}, \quad k \leqslant 6, \\
& C_{2 k}=-\gamma_{k} \sin x_{k-6}^{0} \cos x_{k}^{0}, \quad k>6, \\
& \gamma_{2 k}=0, \quad \forall k \leqslant 6,
\end{aligned}
$$

where $\gamma_{1}=-\gamma_{7}=-\left(2 L_{a}+\Delta L\right), \quad \gamma_{3}=-\gamma_{9}=L_{2}$, and $\gamma_{5}=-\gamma_{11}=$ $-L_{1}$.

\section{The $B$ matrix: Case of differential and common accelerations}

The vector $U$ in Eq. (1) is defined as the components of the given external force $\mathbf{F}$ on the sensitivity plane in the rotating $X^{\prime} Y^{\prime}$ frame, namely, $U \equiv\left\{U_{1}, U_{2}\right\}=\mathbf{F}_{e} \cdot\left\{\hat{X}^{\prime}, \hat{Y}^{\prime}\right\}$. The matrix $B$ transforms the two-component $U$ vector into its $2 n=12$-component counterpart $U(X)$.

In the case of a differential external force, we may figure out $\mathbf{F}_{e}$ as having opposite signs when acting on the two test cylinders. The $B$ matrix is expressed as

$$
\begin{aligned}
& B_{i 1}=\sum_{r=1}^{2 n} \mathrm{M}_{i r} \mathcal{B}_{r 1} \quad \text { for even } i=1, \ldots, 2 n, \\
& B_{i 2}=\sum_{r=1}^{2 n} \mathbb{M}_{i r} \mathcal{B}_{r 2} \quad \text { for even } i=1, \ldots, 2 n,
\end{aligned}
$$

and

$$
B_{i j}=0 \text { for odd } i=1, \ldots, 2 n \text { and } j=1,2 \text {, }
$$

where $\mathbb{M}$ is the "mass" matrix defined in Eq. (29) of Part I. We find

$$
\mathcal{B}=\left(\mathcal{B}_{j 1} \mathcal{B}_{j 2}\right),
$$

where $\mathcal{B}_{j 1}$ is the column vector defined as

$$
\mathcal{B}_{j 1}=\left(\begin{array}{l}
\partial\left[\left(\mathbf{R}_{i}-\mathbf{R}_{o}\right) \cdot \hat{X}^{\prime}\right] / \partial \theta_{a} \\
-\partial\left[\mathbf{R}_{o} \cdot \hat{X}^{\prime}\right] / \partial \theta_{o} \\
+\partial\left[\mathbf{R}_{i} \cdot \hat{X}^{\prime}\right] / \partial \theta_{i} \\
\partial\left[\left(\mathbf{R}_{i}-\mathbf{R}_{o}\right) \cdot \hat{X}^{\prime}\right] / \partial \phi_{a} \\
-\partial\left[\mathbf{R}_{o} \cdot \hat{X}^{\prime}\right] / \partial \phi_{o} \\
+\partial\left[\mathbf{R}_{i} \cdot \hat{X}^{\prime}\right] / \partial \phi_{i}
\end{array}\right),
$$

and $\mathcal{B}_{j 2}$ is obtained from $\mathcal{B}_{j 1}$ after substitution of $\hat{X}^{\prime}$ with $\hat{Y}^{\prime}$.

Common accelerations instead would act on both test bodies and the coupling arm. The resulting $\mathcal{B}$ matrix is then composed by the column vectors,

$$
\mathcal{B}_{j 1}=\left(\begin{array}{l}
\partial\left[\left(\mathbf{R}_{a}\left(m_{a} / m_{i}\right)+\mathbf{R}_{o}+\mathbf{R}_{i}\right) \cdot \hat{X}^{\prime}\right] / \partial \theta_{a} \\
\partial\left[\mathbf{R}_{o} \cdot \hat{X}^{\prime}\right] / \partial \theta_{o} \\
+\partial\left[\mathbf{R}_{i} \cdot \hat{X}^{\prime}\right] / \partial \theta_{i} \\
\partial\left[\left(\mathbf{R}_{a}\left(m_{a} / m_{i}\right)+\mathbf{R}_{o}+\mathbf{R}_{i}\right) \cdot \hat{X}^{\prime}\right] / \partial \phi_{a} \\
-\partial\left[\mathbf{R}_{o} \cdot \hat{X}^{\prime}\right] / \partial \phi_{o} \\
+\partial\left[\mathbf{R}_{i} \cdot \hat{X}^{\prime}\right] / \partial \phi_{i}
\end{array}\right),
$$

and the same for $B_{j 2}$ containing the $Y^{\prime}$ components. In Eq. (A6), the factor $m_{a} / m_{i}$ has been introduced so that the external force produces on the arm the same acceleration as on the inner and outer bodies.

\section{The transfer function in the nonrotating frame}

We show here how to transform the transfer function into the nonrotating frame. In our setting, we may write for the two-component $\left(X^{\prime} Y^{\prime}\right)$ outputs $Y^{\mathrm{NR}}$ and inputs $F^{\mathrm{NR}}$ in the nonrotating frame,

$$
\begin{aligned}
& Y^{\mathrm{NR}}(t)=\mathrm{R}(t) Y(t), \\
& F^{\mathrm{NR}}(t)=\mathrm{R}(t) U(t),
\end{aligned}
$$

where the rotation matrix $\mathbb{R}$ is

$$
R(t)=\left(\begin{array}{cc}
\cos \omega_{s} t & -\sin \omega_{s} t \\
\sin \omega_{s} t & \cos \omega_{s} t
\end{array}\right) .
$$

After introducing the complex variable $Z(t)=Y_{1}(t)+i Y_{2}(t)$, from (A7) and (A9) we have $Z^{\mathrm{NR}}(t)=\exp \left(i \omega_{s} t\right) Z(t)$ and fi- 
nally in the frequency domain $Z^{\mathrm{NR}}(s)=Z\left(s-i \omega_{s}\right)=Y_{1}(s$ $\left.-i \omega_{s}\right)+i Y_{2}\left(s-i \omega_{s}\right)$ or else

$$
Z^{\mathrm{NR}}(s)=\mathfrak{R}\left[Z\left(s-i \omega_{s}\right)\right]+i \mathfrak{I}\left[Z\left(s-i \omega_{s}\right)\right] .
$$

In a similar manner, we also have

$$
W(s)=\mathfrak{R}\left[F^{\mathrm{NR}}\left(s+i \omega_{s}\right)\right]+i \mathfrak{I}\left[F^{\mathrm{NR}}\left(s+i \omega_{s}\right)\right],
$$

with $W(s)=U_{1}(s)+i U_{2}(s)$ and $F^{\mathrm{NR}}(s) \equiv F_{1}^{\mathrm{NR}}(s)+i F_{2}^{\mathrm{NR}}(s)$.

We now evaluate the right-hand side of Eq. (A10) by inserting the expressions of Eq. (3) for $Y_{1}\left(s-i \omega_{s}\right)$ and $Y_{2}(s$ $-i \omega_{s}$ ) and using Eq. (A11) for $W(s)$ (thus $U_{1}$ and $U_{2}$ ). After some simple algebra we obtain

$$
Z^{\mathrm{NR}}(s)=H^{\mathrm{NR}}(s) F^{\mathrm{NR}}(s)
$$

for the nonrotating output $Y^{\mathrm{NR}}(s)$ in response to the nonrotating forces $F^{\mathrm{NR}}$. The non-rotating transfer matrix $H^{\mathrm{NR}}$ turns out to be formed by a combination of the coefficients of the rotating $H$, that is,

$$
H^{\mathrm{NR}}(s)=\left(\begin{array}{cc}
\mathfrak{R}\left[H_{11}\left(s_{-}\right)+i H_{21}\left(s_{-}\right)\right] & \mathfrak{R}\left[H_{12}\left(s_{-}\right)+i H_{22}\left(s_{-}\right)\right] \\
\Im\left[H_{11}\left(s_{-}\right)+i H_{21}\left(s_{-}\right)\right] & \Im\left[H_{12}\left(s_{-}\right)+i H_{22}\left(s_{-}\right)\right]
\end{array}\right),
$$

where we have introduced the shorthand notation $s_{-}$ $\equiv s-i \omega_{s}$.

Let us now look at the poles $p_{l m}^{r}$ and zeros $z_{l m}^{r}$ of the rotor response. The rotating $H_{l m}$ can be expressed as

$$
H_{l m}(s)=\frac{\Pi_{r}\left(s-z_{l m}^{r}+i \omega_{s}\right)\left(s-z_{l m}^{r^{*}}-i \omega_{s}\right)}{\Pi_{r^{\prime}}\left(s-p_{l m}^{r^{\prime}}+i \omega_{s}\right)\left(s-p_{l m}^{r^{\prime *}}-i \omega_{s}\right)},
$$

showing that poles (zeros) in the rotating frame are calculated by shifting the nonspinning values $p_{l m}^{r}\left(z_{l m}^{r}\right)$ by $\pm i \omega_{s}$, namely, $p_{l m}^{r} \pm i \omega_{s}\left(z_{l m}^{r} \pm i \omega_{s}\right)$. By inspection from Eq. (A13), it follows that the poles (zeros) of $H^{\mathrm{NR}}$ in the nonrotating frame can be expressed in terms of the $p_{l m}^{r}\left(z_{l m}^{r}\right)$ nonspinning values as combinations of a dc component $p_{l m}^{r}$ and of a term $p_{l m}^{r}$ $+2 i \omega_{2}$ modulated at twice the spin frequency $\left(z_{l m}^{r}\right.$ and $z_{l m}^{r}$ $\left.+2 i \omega_{2}\right)$.

${ }^{1}$ E. Fischbach, D. E. Krause, C. Talmadge, and D. Tadic, Phys. Rev. D 52, 5417 (1995).

${ }^{2}$ T. Damour, F. Piazza, and G. Veneziano, Phys. Rev. Lett. 89, 081601 (2002).

${ }^{3}$ Y. Su et al., Phys. Rev. D 50, 3614 (1994).

${ }^{4}$ S. Baebler, B. R. Heckel, E. G. Adelberger, J. H. Gundlach, U. Schimidt, and H. E. Swanson, Phys. Rev. Lett. 83, 3585 (1999).

${ }^{5}$ P. W. Worden Jr. and C. W. F. Everitt, in Experimental Gravitation, Proceedings of the "Enrico Fermi" International School of Physics, Course LVI, edited by B. Bertotti (Academic, New York, 1973); J. P. Blaser et al., ESA SCI Report No. (96)5, 1996 (unpublished); see also the STEP website http://einstein.stanford.edu/STEP/step2.html

${ }^{6}$ See the MICROSCOPE website http://www.onera.fr/dmph/accelerometre/ index.html

${ }^{7}$ A. M. Nobili, D. Bramanti, G. L. Comandi, R. Toncelli, E. Polacco, and M. L. Chiofalo, Phys. Lett. A 318, 172 (2003); Galileo Galilei (GG) Phase A Report, ASI, 2nd ed., January 2000; A. M. Nobili, D. Bramanti, G. Comandi, R. Toncelli, E. Polacco, and G. Catastini, Phys. Rev. D 63, 101101 (2001); for a review see, e.g., A. Nobili, in Recent Advances in Metrology and Fundamental Constants, Proceedings of the "Enrico Fermi" International School of Physics, Course CXLVI, edited by T. J. Quinn, S. Leschiutta, and P. Tavella (IOS, IOS Press, 2001), p. 609; see also the GG website http://eotvos.dm.unipi.it/nobili

${ }^{8}$ A. M. Nobili, D. Bramanti, G. L. Comandi, R. Toncelli, and E. Polacco, New Astron. Rev. 8, 371 (2003).

${ }^{9}$ G. L. Comandi, A. M. Nobili, D. Bramanti, R. Toncelli, E. Polacco, and M. L. Chiofalo, Phys. Lett. A 318, 213 (2003).

${ }^{10}$ G. L. Comandi, A. M. Nobili, R. Toncelli, and M. L. Chiofalo, Phys. Lett. A 318, 251 (2003).

${ }^{11}$ A. M. Nobili, D. Bramanti, G. L. Comandi, R. Toncelli, E. Polacco, and M. L. Chiofalo, in Proceedings of the XXXVIIIth Rencontre de Moriond "Gravitational Waves and Experimental Gravity," edited by J. Dumarchez and J. Tran Thanh Van (The Gioi, Vietnam, 2003), p. 371.

${ }^{12}$ Note that $K_{i}$ and $K_{o}$ are bound to be very similar to $K$ for symmetry reasons, and that $L_{o}$ is related to $L_{i}$ through $L_{o}=2 L_{a}+\Delta L+L_{i}$. Thus $L_{i}$ (or else $L_{o}$ ) is the remaining independent parameter. 